\title{
Two Types of Solutions to a Class of $(p, q)$-Laplacian Systems with Critical Sobolev Exponents in $\mathbb{R}^{\mathbb{N}}$
}

\author{
Jing Li ${ }^{1}{ }^{1,2}$ and Caisheng Chen (iD) \\ ${ }^{1}$ College of Science, Hohai University, Nanjing 210098, China
}

${ }^{2}$ College of Science, Linyi University, Linyi 276005, China

Correspondence should be addressed to Jing Li; slyphna@163.com

Received 11 October 2017; Accepted 12 December 2017; Published 29 January 2018

Academic Editor: Ming Mei

Copyright ( 2018 Jing Li and Caisheng Chen. This is an open access article distributed under the Creative Commons Attribution License, which permits unrestricted use, distribution, and reproduction in any medium, provided the original work is properly cited.

We focus on the following elliptic system with critical Sobolev exponents: $-\operatorname{div}\left(|\nabla u|^{p-2} \nabla u\right)+m(x)|u|^{p-2} u=\lambda|u|^{p^{*}-2} u+$ $(1 / \eta) G_{u}(u, v), \quad x \in \mathbb{R}^{N} ;-\operatorname{div}\left(|\nabla v|^{q-2} \nabla v\right)+n(x)|v|^{q-2} v=\mu|v|^{q^{*}-2} v+(1 / \eta) G_{v}(u, v), x \in \mathbb{R}^{N} ; u(x)>0, v(x)>0, x \in \mathbb{R}^{N}$, where $\mu, \lambda>0,1<p \leq q<N$, either $\eta \in(1, p)$ or $\eta \in\left(q, p^{*}\right)$, and critical Sobolev exponents $p^{*}=p N /(N-p)$ and $q^{*}=q N /(N-q)$. Conditions on potential functions $m(x), n(x)$ lead to no compact embedding. Relying on concentration-compactness principle, mountain pass lemma, and genus theory, the existence of solutions to the elliptic system with $\eta \in\left(q, p^{*}\right)$ or $\eta \in(1, p)$ will be established.

\section{Introduction and Main Result}

We aim at the existence of nontrivial weak solutions for the following elliptic system:

$$
\begin{aligned}
& -\operatorname{div}\left(|\nabla u|^{p-2} \nabla u\right)+m(x)|u|^{p-2} u \\
& =\lambda|u|^{p^{*}-2} u+\frac{1}{\eta} G_{u}(u, v), \quad x \in \mathbb{R}^{N}, \\
& -\operatorname{div}\left(|\nabla v|^{q-2} \nabla v\right)+n(x)|v|^{q-2} v \\
& =\mu|v|^{q^{*}-2} v+\frac{1}{\eta} G_{v}(u, v), \quad x \in \mathbb{R}^{N}, \\
& u(x)>0, \quad v(x)>0, \quad x \in \mathbb{R}^{N},
\end{aligned}
$$

where $\mu, \lambda>0,1<p \leq q<p^{*}<q^{*}$, and either $1<\eta<p$ or $q<\eta<p^{*}$.

Discussion regarding critical growth has been dominated research in various branches of mathematical physics. Here, we quote a few literatures on critical Sobolev exponent and their applications; see Brezis and Nirenberg [1], Wu [2-4], and Garcia-Azorero et al. [5] and the references therein.
By applying variational arguments, Yin and Yang in [6] proved the existence of at least one or two positive solutions to the following system:

$$
\begin{aligned}
& -\Delta_{p} u+|u|^{p-2} u=f_{1 \lambda_{1}}(x)|u|^{q^{*}-2} u \\
& +\frac{2 \alpha}{\alpha+\beta} g_{\mu}(x)|u|^{\alpha-2} u|v|^{\beta}, \\
& -\Delta_{p} v+|v|^{p-2} v=f_{2 \lambda_{2}}(x)|v|^{q^{*}-2} v \\
& +\frac{2 \beta}{\alpha+\beta} g_{\mu}(x)|u|^{\alpha}|v|^{\beta-2} v, \\
& x \in \Omega \text {, } \\
& x \in \Omega \text {, } \\
& u=v=0, \quad \text { on } \partial \Omega,
\end{aligned}
$$

where $F_{i \lambda_{i}}(x)=\lambda_{i} f_{i_{+}}(x)+f_{i_{-}}(x)(i=1,2)$ are sign-changing functions.

Subsequently, at least one nontrivial solution was established in the work [7]: 


$$
\begin{aligned}
-\Delta_{p} u & =f(x)|u|^{p^{*}-2} u+\lambda \frac{\partial F}{\partial u}(x, u, v), \quad \text { in } \mathbb{R}^{N}, \\
-\Delta_{q} v & =g(x)|v|^{q^{*}-2} v+\lambda \frac{\partial F}{\partial v}(x, u, v), \quad \text { in } \mathbb{R}^{N}, \\
u(x), v(x) & \longrightarrow 0, \quad \text { as }|x| \longrightarrow \infty
\end{aligned}
$$

via mountain pass lemma. More results can be found in [710].

In papers cited above, the authors are fond of supposing that potential functions $m(x), n(x)$ satisfy such conditions:

$\left(\mathscr{F}_{1}\right)$ Functions $m(x), n(x) \in C\left(\mathbb{R}^{N}\right)$ and satisfy $m(x)$, $n(x) \geq c_{0}>0$ with some constant $c_{0}$. Moreover, for every $\kappa>0$, meas $\left(\left\{x \in \mathbb{R}^{N}: m(x), n(x) \leq \kappa\right\}\right)<\infty$, where "meas" denotes the Lebesgue measure in $\mathbb{R}^{N}$.

$\left(\mathscr{F}_{2}\right) m(x) \rightarrow+\infty, n(x) \rightarrow+\infty$, as $n \rightarrow \infty$.

We point out that one of the above conditions has been used in many papers to guarantee the compact embedding $W^{1, p}\left(\mathbb{R}^{N}\right) \hookrightarrow L^{\nu}\left(\mathbb{R}^{N}\right)$ for each $v \in\left[p, p^{*}\right)$. Nevertheless, throughout the paper, we are going to weaken the conditions and give assumptions as follows:

$\left(\mathscr{H}_{1}\right)$ Let nonnegative function $G(u, v) \in C^{1}\left(\mathbb{R}^{2}\right)$ be homogeneous with $G(t u, t v)=t^{\eta} G(u, v)$, where $\forall(u, v) \epsilon$ $\mathbb{R}^{2}$ and either $\eta \in(1, p)$ or $\eta \in\left(q, p^{*}\right)$. Furthermore, there exists $\beta(x)>0$ such that

$$
\begin{aligned}
0 & \leq G(u, v), G_{u}(u, v) u, G_{v}(u, v) v \\
& \leq \beta(x)\left(|u|^{\eta}+|v|^{\eta}\right), \quad \forall(u, v) \in \mathbb{R}^{2},
\end{aligned}
$$

and for all $(u, v),(\xi, \zeta) \in \mathbb{R}^{2}$

$$
\begin{aligned}
& |G(u, v)-G(\xi, \zeta)| \leq \beta(x) \\
& \cdot\left(|u|^{\eta-1}+|v|^{\eta-1}+|\xi|^{\eta-1}+|\zeta|^{\eta-1}\right) \\
& \cdot(|u-\xi|+|v-\zeta|) .
\end{aligned}
$$

$\left(\mathscr{H}_{2}\right)$ Functions $m(x), n(x) \in C\left(\mathbb{R}^{N}\right)$ and satisfy $m(x)$, $n(x) \geq c_{0}$ with some constant $c_{0}$. In addition, for every $\kappa>0$, meas $\left(\left\{x \in \mathbb{R}^{N}: m(x) / \beta(x), n(x) / \beta(x) \leq \kappa\right\}\right)<$ $\infty$, where "meas" denotes the Lebesgue measure in $\mathbb{R}^{N}$.

$\left(\mathscr{H}_{3}\right)$ Function $\beta(x)$ is positive and $\beta(x) \in L_{\text {loc }}^{\sigma}\left(\mathbb{R}^{N}\right)$ with $\sigma=p^{*} /\left(p^{*}-\eta\right), \eta \in\left(p, q^{*}\right)$. Moreover, $\lim \sup _{|x| \rightarrow \infty} \beta(x)<\infty$.

$\left(\mathscr{H}_{4}\right)$ Function $\beta(x)$ is positive and $\beta(x) \in L^{\gamma}\left(\mathbb{R}^{N}\right)$ with $\gamma=$ $q^{*} /\left(q^{*}-\eta\right), \eta \in(1, p)$.

Condition $\left(\mathscr{H}_{2}\right)$ used in our paper is weaker than $\left(\mathscr{F}_{1}\right)$ which leads to no compact embedding of $W^{1, p}\left(\mathbb{R}^{N}\right) \hookrightarrow L^{\nu}\left(\mathbb{R}^{N}\right)$. As far as the authors are aware, there is less literature dealing with such potential functions. Moreover, a more generalized function $G(u, v)$ appeared in the paper.
In order to state our main result, let us recall some Sobolev spaces and norms. Let $X, Y$ be subspaces of $W^{1, p}\left(\mathbb{R}^{N}\right)$, $W^{1, q}\left(\mathbb{R}^{N}\right)$ with the norms

$$
\begin{aligned}
& X=\left\{u \in W^{1, p}\left(\mathbb{R}^{N}\right): \int_{\mathbb{R}^{N}} m(x)|u|^{p} d x<\infty\right\} \\
& \text { with }\|u\|_{X}=\left(\int_{\mathbb{R}^{N}}\left(|\nabla u|^{p}+m(x)|u|^{p}\right) d x\right)^{1 / p}, \\
& Y=\left\{v \in W^{1, q}\left(\mathbb{R}^{N}\right): \int_{\mathbb{R}^{N}} n(x)|v|^{q} d x<\infty\right\} \\
& \text { with }\|v\|_{Y}=\left(\int_{\mathbb{R}^{N}}\left(|\nabla v|^{q}+n(x)|v|^{q}\right) d x\right)^{1 / q}, \\
& \|u\|_{t}=\left(\int_{\mathbb{R}^{N}}|u|^{t} d x\right)^{1 / t}, \\
& \|u\|_{t, m}=\left(\int_{\mathbb{R}^{N}} m(x)|u(x)|^{t} d x\right)^{1 / t}, \\
& \|u\|_{t, n}=\left(\int_{\mathbb{R}^{N}} n(x)|u(x)|^{t} d x\right)^{1 / t},
\end{aligned}
$$

$$
1<t<N \text {. }
$$

Obviously, the norm of reflexive Banach space $E=X \times Y$ is given by

$$
\|(u, v)\|_{E}=\|u\|_{X}+\|v\|_{Y}, \quad \forall(u, v) \in E .
$$

Define

$$
\begin{aligned}
& S_{1}=\inf _{u \in X \backslash\{0\}} \frac{\|u\|_{X}^{p}}{\left(\int_{\mathbb{R}^{N}}|u|^{p^{*}}\right)^{p / p^{*}}}, \\
& S_{2}=\inf _{v \in Y \backslash\{0\}} \frac{\|v\|_{Y}^{q}}{\left(\int_{\mathbb{R}^{N}}|v|^{q^{*}}\right)^{q / q^{*}}}, \\
& c^{*}=\min \left\{\frac{\lambda}{N}\left(\frac{S_{1}}{\lambda}\right)^{N / P}, \frac{\mu}{N}\left(\frac{S_{2}}{\mu}\right)^{N / q}\right\} .
\end{aligned}
$$

Corresponding functional of (1) defined in $E$ is

$$
\begin{aligned}
J(u, v)= & \frac{1}{p}\|u\|_{X}^{p}+\frac{1}{q}\|v\|_{Y}^{q}-\frac{\lambda}{p^{*}} \int_{\mathbb{R}^{N}}|u|^{p^{*}} d x \\
& -\frac{\mu}{q^{*}} \int_{\mathbb{R}^{N}}|v|^{q^{*}} d x-\frac{1}{\eta} \int_{\mathbb{R}^{N}} G(u, v) d x .
\end{aligned}
$$

The main results are enunciated as follows.

Theorem 1. Suppose $\left(\mathscr{H}_{1}\right),\left(\mathscr{H}_{2}\right)$, and $\left(\mathscr{H}_{3}\right)$ with $\eta \in\left(q, p^{*}\right)$ hold; then there exists $\Lambda_{0}>0$ such that if $0<\lambda, \mu<\Lambda_{0}$, system (1) has at least a nontrivial solution. 
Theorem 2. Suppose $\left(\mathscr{H}_{1}\right),\left(\mathscr{H}_{2}\right)$, and $\left(\mathscr{H}_{4}\right)$ with $\eta \in(1, p)$ hold; then there exists $\Lambda_{1}>0$ such that if $0<\lambda, \mu<\Lambda_{1}$, problem (1) has infinitely many solutions $\left(u_{n}, v_{n}\right) \in E$ with $J\left(u_{n}, v_{n}\right)<0, J\left(u_{n}, v_{n}\right) \rightarrow 0$, and $\left(u_{n}, v_{n}\right) \rightarrow 0$ as $n \rightarrow \infty$.

This work is structured as follows. Variational framework will be set up and some useful lemmas will be derived in Section 2. The proofs of Theorems 1 and 2 are established in Sections 3 and 4, respectively.

\section{Preliminaries}

The main purpose of this section is to present that functional $J$ satisfies $(P S)_{c}$ condition. Besides, some useful technical lemmas will be listed.

Lemma 3. Assume that $\left(\mathscr{H}_{1}\right)-\left(\mathscr{H}_{4}\right)$ hold; if $\left\{\left(u_{n}, v_{n}\right)\right\} \subset E$ is $a(P S)_{c}$ sequence, then sequence $\left\{\left(u_{n}, v_{n}\right)\right\}$ is bounded in $E$.

Proof. Let $J\left(u_{n}, v_{n}\right) \rightarrow c, J^{\prime}\left(u_{n}, v_{n}\right) \rightarrow 0$ in $E^{*}$ as $n \rightarrow \infty$.

Case 1. When $\eta \in\left(q, p^{*}\right)$, we can choose $q<\theta<\eta$ such that

$$
\begin{aligned}
c+1 & +\left\|\left(u_{n}, v_{n}\right)\right\|_{E} \geq J\left(u_{n}, v_{n}\right)-\frac{1}{\theta} J^{\prime}\left(u_{n}, v_{n}\right)\left(u_{n}, v_{n}\right) \\
= & \left(\frac{1}{p}-\frac{1}{\theta}\right)\left\|u_{n}\right\|_{X}^{p}+\left(\frac{1}{q}-\frac{1}{\theta}\right)\left\|v_{n}\right\|_{Y}^{q} \\
& +\left(\frac{1}{\theta}-\frac{1}{p^{*}}\right) \int_{\mathbb{R}^{N}} \lambda\left|u_{n}\right|^{p^{*}} d x \\
& +\left(\frac{1}{\theta}-\frac{1}{q^{*}}\right) \int_{\mathbb{R}^{N}} \mu\left|v_{n}\right|^{q^{*}} d x \\
& +\left(\frac{1}{\theta}-\frac{1}{\eta}\right) \int_{\mathbb{R}^{N}} G\left(u_{n}, v_{n}\right) d x \\
\geq & \left(\frac{1}{p}-\frac{1}{\theta}\right)\left\|u_{n}\right\|_{X}^{p}+\left(\frac{1}{q}-\frac{1}{\theta}\right)\left\|v_{n}\right\|_{Y}^{q} \\
\geq & \left(\frac{1}{q}-\frac{1}{\theta}\right) \min \left\{\left\|\left(u_{n}, v_{n}\right)\right\|_{E}^{p},\left\|\left(u_{n}, v_{n}\right)\right\|_{E}^{q}\right\} .
\end{aligned}
$$

Case 2. When $\eta \in(1, p)$, we can choose $q<\theta<p^{*}$ such that

$$
\begin{aligned}
c+ & 1+\left\|\left(u_{n}, v_{n}\right)\right\|_{E} \geq J\left(u_{n}, v_{n}\right)-\frac{1}{\theta} J^{\prime}\left(u_{n}, v_{n}\right)\left(u_{n}, v_{n}\right) \\
& =\left(\frac{1}{p}-\frac{1}{\theta}\right)\left\|u_{n}\right\|_{X}^{p}+\left(\frac{1}{q}-\frac{1}{\theta}\right)\left\|v_{n}\right\|_{Y}^{q}+\left(\frac{1}{\theta}-\frac{1}{p^{*}}\right) \\
& \cdot \int_{\mathbb{R}^{N}} \lambda\left|u_{n}\right|^{p^{*}} d x+\left(\frac{1}{\theta}-\frac{1}{q^{*}}\right) \int_{\mathbb{R}^{N}} \mu\left|v_{n}\right|^{q^{*}} d x \\
& -\left(\frac{1}{\eta}-\frac{1}{\theta}\right) \int_{\mathbb{R}^{N}} G\left(u_{n}, v_{n}\right) d x \geq\left(\frac{1}{p}-\frac{1}{\theta}\right)\left\|u_{n}\right\|_{X}^{p} \\
& +\left(\frac{1}{q}-\frac{1}{\theta}\right)\left\|v_{n}\right\|_{Y}^{q}-\left(\frac{1}{\eta}-\frac{1}{\theta}\right) \int_{\mathbb{R}^{N}} \beta(x)\left(\left|u_{n}\right|^{\eta}\right. \\
& \left.+\left|v_{n}\right|^{\eta}\right) d x \geq\left(\frac{1}{q}-\frac{1}{\theta}\right)
\end{aligned}
$$

$$
\begin{aligned}
& \cdot \min \left\{\left\|\left(u_{n}, v_{n}\right)\right\|_{E}^{p},\left\|\left(u_{n}, v_{n}\right)\right\|_{E}^{q}\right\}-\left(\frac{1}{\eta}-\frac{1}{\theta}\right)\|\beta(x)\|_{\vartheta} \\
& \cdot\left\|u_{n}\right\|_{p^{*}}^{\eta}-\left(\frac{1}{\eta}-\frac{1}{\theta}\right)\|\beta(x)\|_{\gamma}\left\|v_{n}\right\|_{q^{*}}^{\eta} \geq\left(\frac{1}{q}-\frac{1}{\theta}\right) \\
& \cdot \min \left\{\left\|\left(u_{n}, v_{n}\right)\right\|_{E}^{p},\left\|\left(u_{n}, v_{n}\right)\right\|_{E}^{q}\right\}-\left(\frac{1}{\eta}-\frac{1}{\theta}\right) \\
& \cdot S_{1}^{-\eta / p}\|\beta(x)\|_{\vartheta}\left\|u_{n}\right\|_{X}^{\eta}-\left(\frac{1}{\eta}-\frac{1}{\theta}\right) S_{2}^{-\eta / q}\|\beta(x)\|_{\gamma} \\
& \cdot\left\|v_{n}\right\|_{Y}^{\eta} \geq\left(\frac{1}{q}-\frac{1}{\theta}\right) \min \left\{\left\|\left(u_{n}, v_{n}\right)\right\|_{E}^{p},\left\|\left(u_{n}, v_{n}\right)\right\|_{E}^{q}\right\} \\
& -\max \left\{\left(\frac{1}{\eta}-\frac{1}{\theta}\right) S_{1}^{-\eta / p}\|\beta(x)\|_{\vartheta},\left(\frac{1}{\eta}-\frac{1}{\theta}\right)\right. \\
& \left.\cdot S_{2}^{-\eta / q}\|\beta(x)\|_{\gamma}\right\}\left\|\left(u_{n}, v_{n}\right)\right\|_{E}^{\eta},
\end{aligned}
$$

where $\vartheta=p^{*} /\left(p^{*}-\eta\right) \geq \gamma=q^{*} /\left(q^{*}-\eta\right)$; the above inequalities imply the sequence $\left\{\left(u_{n}, v_{n}\right)\right\}$ is bounded in $E$ and the proof is finished.

As the consequence of Lemma 3, there exist a constant $M>0,(u, v) \in E$, and sequence $\left\{\left(u_{n}, v_{n}\right)\right\} \in E$ such that

$$
\begin{aligned}
\left\|\left(u_{n}, v_{n}\right)\right\|_{E} \leq M, \quad \forall n \in N,\|(u, v)\|_{E} \leq M \\
\left(u_{n}, v_{n}\right) \rightarrow(u, v) \text { weakly in } E, \\
\left(u_{n}(x), v_{n}(x)\right) \longrightarrow(u(x), v(x)) \text { a.e. in } \mathbb{R}^{N} \\
\left(u_{n}, v_{n}\right) \longrightarrow(u, v) \text { strongly in } L_{\text {loc }}^{s}\left(\mathbb{R}^{N}\right) \\
\times L_{\text {loc }}^{r}\left(\mathbb{R}^{N}\right), \\
\forall s \in\left[1, p^{*}\right), r \in\left[1, q^{*}\right) .
\end{aligned}
$$

Lemma 4. Let $\left(\mathscr{H}_{1}\right),\left(\mathscr{H}_{2}\right)$, and $\left(\mathscr{H}_{3}\right)$ be satisfied with $p \leq \eta<$ $p^{*}$. Suppose sequence $\left\{u_{n}\right\}$ is bounded in $X$; then there exists $u \in X \cap L^{\eta}\left(\mathbb{R}^{N}, \beta\right)$ such that $u_{n} \rightarrow u$ strongly in $L^{\eta}\left(\mathbb{R}^{N}, \beta\right)$.

Proof. Since $\left\{u_{n}\right\}$ is bounded in $X$, there exist $u \in X$ and $M_{1}>$ 0 such that

$$
\begin{aligned}
\left\|u_{n}\right\|+\|u\| \leq M_{1} ; & \\
\qquad u_{n} \longrightarrow u \text { strongly in } L_{\text {loc }}^{s}\left(\mathbb{R}^{N}\right), & \\
& \forall s \in\left[1, p^{*}\right) .
\end{aligned}
$$

Thus, we have that $u_{n} \rightarrow u$ strongly in $L^{p}\left(B_{i}, \beta\right)$, where $B_{i}=$ $\left\{x \in \mathbb{R}^{N}:|x|<i\right\}$ and $B_{i}^{c}=\left\{x \in \mathbb{R}^{N}:|x| \geq i\right\}$.

Step 1. We want to show that, for $\forall \epsilon>0$, there exists $R>1$ such that

$$
\int_{B_{R}^{c}} \beta(x)\left|u_{n}\right|^{p} d x<\epsilon .
$$


We can fix $\epsilon>0$ and choose $t \in(1, N /(N-p))$ and $a_{0}, \kappa$, where

$$
\begin{aligned}
& a_{0}=\sup _{X \backslash\{0\}} \frac{\left\|u_{n}\right\|_{p t}^{p}}{\left\|u_{n}\right\|_{X}^{p}}, \\
& \kappa>\frac{2 M_{1}^{p}}{\epsilon} \geq \frac{2}{\epsilon} \sup _{n \geq 1}\left\|u_{n}\right\|_{X}^{p} .
\end{aligned}
$$

Denote $A_{i}=\left\{x \in B_{i}: m(x) / \beta(x) \leq \kappa\right\}$; then $A_{i} \subset A_{i+1}$ for $i=1,2, \ldots$, and $A_{\infty}=\left\{x \in \mathbb{R}^{N}: m(x) / \beta(x) \leq \kappa\right\}=$ $\bigcup_{i=1}^{\infty} A_{i}$. What is more, there exist $i_{0} \geq 1$ and $\beta_{0}>0$ such that $|\beta(x)| \leq \beta_{0}$ for $|x| \geq i_{0}$ by condition $\left(\mathscr{H}_{3}\right)$ and meas $\left(A_{\infty}\right)$ is bounded by condition $\left(\mathscr{H}_{2}\right)$; then, for every $\epsilon>0$, there exists $i_{1} \geq i_{0}$ such that for $i \geq i_{1}$

$$
\begin{aligned}
0 & \leq \text { meas }\left(A_{\infty}\right)-\operatorname{meas}\left(A_{i}\right)=\operatorname{meas}\left(\Omega_{i}^{c}\right) \\
& \leq\left(\frac{\epsilon}{2 a_{0} \beta_{0} M_{1}^{p}}\right)^{t /(t-1)},
\end{aligned}
$$

where

$$
\begin{aligned}
& \Omega_{i}=\left\{x \in B_{i}^{c}: \frac{m(x)}{\beta(x)}>\kappa\right\}, \\
& \Omega_{i}^{c}=\left\{x \in B_{i}^{c}: \frac{m(x)}{\beta(x)} \leq \kappa\right\} .
\end{aligned}
$$

By $(15)$ and $\left(\mathscr{H}_{2}\right)$, we observe that

$$
\begin{aligned}
& \int_{\Omega_{i}} \beta(x)\left|u_{n}\right|^{p} d x \leq \frac{1}{\kappa} \int_{\Omega_{i}} m(x)\left|u_{n}\right|^{p} d x \\
& \quad \leq \frac{1}{\mathcal{\kappa}} \int_{\mathbb{R}^{N}} m(x)\left|u_{n}\right|^{p} d x \leq \frac{1}{\kappa}\left\|u_{n}\right\|_{X}^{p} \leq \frac{\epsilon}{2}, \\
& \int_{\Omega_{i}^{c}} \beta(x)\left|u_{n}\right|^{p} d x \\
& \quad \leq\left(\int_{\Omega_{i}^{c}}\left|u_{n}\right|^{p t} d x\right)^{1 / t}\left(\int_{\Omega_{i}^{c}}|\beta(x)|^{t /(t-1)} d x\right)^{(t-1) / t} \\
& \leq a_{0}\left\|u_{n}\right\|_{X}^{p}\|\beta(x)\|_{L^{t /(t-1)}\left(\Omega_{i}^{c}\right)} \\
& \leq a_{0} \beta_{0}\left\|u_{n}\right\|_{X}^{p}\left[\operatorname{meas}\left(\Omega_{i}^{c}\right)\right]^{(t-1) / t} \leq \frac{\epsilon}{2} .
\end{aligned}
$$

Therefore, we get

$$
\begin{aligned}
\int_{B_{i}^{c}} \beta(x)\left|u_{n}\right|^{p} d x= & \int_{\Omega_{i}} \beta(x)\left|u_{n}\right|^{p} d x \\
& +\int_{\Omega_{i}^{c}} \beta(x)\left|u_{n}\right|^{p} d x \leq \epsilon,
\end{aligned}
$$

and by Fatou's lemma

$$
\int_{B_{i}^{c}} \beta(x)|u|^{p} d x \leq \liminf _{n \rightarrow \infty} \int_{B_{i}^{c}} \beta(x)\left|u_{n}\right|^{p} d x \leq \epsilon .
$$

Thus, from (13) and (20), we can get that $u_{n} \rightarrow u$ strongly in $L^{p}\left(\mathbb{R}^{N}, \beta\right)$.

Step 2. For $p<\eta<p^{*}$, let $\eta=r p+(1-r) p^{*}, r \in(0,1)$. By (19), we have that

$$
\begin{aligned}
& \int_{B_{i}^{c}} \beta(x)\left|u_{n}\right|^{\eta} d x \\
& \quad \leq \int_{B_{i}^{c}} \beta(x)^{r}\left|u_{n}\right|^{r p} \beta(x)^{1-r}\left|u_{n}\right|^{(1-r) p^{*}} d x \\
& \quad \leq \beta_{0}^{1-r}\left\|u_{n}\right\|_{L^{p}\left(B_{i}^{c}, \beta\right)}^{r p}\left\|u_{n}\right\|_{L^{p^{*}}\left(B_{i}^{c}\right)}^{(1-r) p^{*}} \\
& \quad \leq \beta_{0}^{1-r} S_{1}^{-(1-r) p^{*} / p}\left\|u_{n}\right\|_{X}^{(1-r) p^{*}}\left\|u_{n}\right\|_{L^{p}\left(B_{i}^{c}, \beta\right)}^{r p} \\
& \leq \beta_{0}^{1-r} S_{1}^{-(1-r) p^{*} / p} M_{1}^{(1-r) p^{*}} \epsilon=C \epsilon .
\end{aligned}
$$

By Fatou's lemma, we can see that

$\int_{B_{i}^{c}} \beta(x)|u|^{\eta} d x \leq \lim \inf _{n \rightarrow \infty} \int_{B_{i}^{c}} \beta(x)\left|u_{n}\right|^{\eta} d x \leq C \epsilon$.

For each measure subset $\Omega \subset B_{i}$, we have that

$$
\begin{aligned}
\int_{\Omega} \beta(x)\left|u_{n}-u\right|^{\eta} d x & \leq\|\beta\|_{L^{\sigma}(\Omega)}\left\|u_{n}-u\right\|_{L^{p^{*}}(\Omega)}^{\eta} \\
& \leq\|\beta\|_{L^{\sigma}(\Omega)}\left\|u_{n}-u\right\|_{L^{p^{*}}\left(\mathbb{R}^{N}\right)}^{\eta} \\
& \leq S_{1}^{-\eta / p}\left(2 M_{1}\right)^{\eta}\|\beta\|_{L^{\sigma}(\Omega)} .
\end{aligned}
$$

Thus, one can get that $\left\{\beta(x)\left|u_{n}-u\right|^{\eta}\right\}$ is uniformly integral and $\int_{B_{i}} \beta(x)\left|u_{n}-u\right|^{\eta} d x$ is bounded. Then, we have that

$$
\lim _{n \rightarrow \infty} \int_{B_{i}} \beta(x)\left|u_{n}-u\right|^{\eta} d x=0
$$

Therefore, from (22) and (24), we can obtain

$$
\lim _{n \rightarrow \infty} \int_{\mathbb{R}^{N}} \beta(x)\left|u_{n}-u\right|^{\eta} d x=0 .
$$

Similar argument can be applied to get that $v_{n} \rightarrow$ $v$ strongly in $L^{\eta}\left(\mathbb{R}^{N}, \beta\right)$. Thus, one can easily get that $\lim _{n \rightarrow \infty} \int_{\mathbb{R}^{N}} G\left(u_{n}, v_{n}\right) d x=\int_{\mathbb{R}^{N}} G(u, v) d x$.

Lemma 5. Assume that $\left(\mathscr{H}_{1}\right)$ and $\left(\mathscr{H}_{4}\right)$ hold with $\eta \in(1, p)$; if $\left\{\left(u_{n}, v_{n}\right)\right\}$ satisfies (12), then

$$
\lim _{n \rightarrow \infty} \int_{\mathbb{R}^{N}} G\left(u_{n}, v_{n}\right) d x=\int_{\mathbb{R}^{N}} G(u, v) d x .
$$

Proof. By $\left(\mathscr{H}_{1}\right)$ and $\left(\mathscr{H}_{4}\right)$, we get that

$$
\int_{B_{R}} \beta(x)\left|u_{n}\right|^{\eta} d x \longrightarrow \int_{B_{R}} \beta(x)|u|^{\eta} d x, \quad n \longrightarrow \infty,
$$


where $B_{R}=\left\{x \in \mathbb{R}^{N}:|x|<R\right\}$ and $B_{R}^{c}=\mathbb{R}^{N} \backslash \overline{B_{R}}$ for any $R>0$. Moreover, we obtain from Hölder inequality that

$$
\begin{aligned}
& \int_{B_{R}^{c}}|\beta(x)|\left|u_{n}\right|^{\eta} d x \\
& \leq\left(\int_{B_{R}^{c}}|\beta(x)|^{p^{*} /\left(p^{*}-\eta\right)}\right)^{\left(p^{*}-\eta\right) / p^{*}}\left(\int_{B_{R}^{c}}\left|u_{n}\right|^{p^{*}}\right)^{\eta / p^{*}} \\
& \leq S_{1}^{-\eta / p}\|\beta(x)\|_{L^{9}\left(B_{R}^{c}\right)}\left\|u_{n}\right\|_{E}^{\eta} \\
& \leq S_{1}^{-\eta / p} M^{\eta}\|\beta(x)\|_{L^{9}\left(B_{R}^{c}\right)} \longrightarrow 0, \quad \text { as } R \longrightarrow \infty,
\end{aligned}
$$

where $\vartheta=p^{*} /\left(p^{*}-\eta\right) \geq \gamma=q^{*} /\left(q^{*}-\eta\right)$. By Fatou's lemma, we have

$$
\begin{aligned}
\int_{B_{R}^{c}}|\beta(x)||u|^{\eta} d x & \leq \lim _{n \rightarrow \infty} \int_{B_{R}^{c}}|\beta(x)|\left|u_{n}\right|^{\eta} d x \\
\leq M^{\eta}\|\beta(x)\|_{L^{\gamma}\left(B_{R}^{c}\right)} \longrightarrow & 0, \\
& \text { as } R \longrightarrow \infty .
\end{aligned}
$$

Then, from (28) and (29), we can deduce $\int_{\mathbb{R}^{N}} \beta(x)\left|u_{n}\right|^{\eta} d x \rightarrow$ $\int_{\mathbb{R}^{N}} \beta(x)|u|^{\eta} d x$. Similarly, we can get $\int_{\mathbb{R}^{N}} \beta(x)\left|v_{n}\right|^{\eta} d x \rightarrow$ $\int_{\mathbb{R}^{N}} \beta(x)|v|^{\eta} d x$. Thus, the proof is completed.

$$
\begin{aligned}
& \text { Denote } \\
& \begin{aligned}
P_{n} & =J^{\prime}\left(u_{n}, v_{n}\right)\left(u_{n}-u, v_{n}-v\right) \\
& =\int_{\mathbb{R}^{N}}\left[\left|\nabla u_{n}\right|^{p-2} \nabla u_{n} \nabla\left(u_{n}-u\right)\right. \\
& \left.+m(x)\left|u_{n}\right|^{p-2} u_{n}\left(u_{n}-u\right)\right] d x \\
& +\int_{\mathbb{R}^{N}}\left[\left|\nabla v_{n}\right|^{q-2} \nabla v_{n} \nabla\left(v_{n}-v\right)\right. \\
& \left.+n(x)\left|v_{n}\right|^{q-2} v_{n}\left(v_{n}-u\right)\right] d x \\
& -\lambda \int_{\mathbb{R}^{N}}\left|u_{n}\right|^{p^{*}-2} u_{n}\left(u_{n}-u\right) d x-\mu \int_{\mathbb{R}^{N}}\left|v_{n}\right|^{q^{*}-2} \\
& \cdot v_{n}\left(v_{n}-v\right) d x-\frac{1}{\eta} \\
& \cdot \int_{\mathbb{R}^{N}}\left[G_{u}\left(u_{n}, v_{n}\right)\left(u_{n}-u\right)\right. \\
& \left.+G_{v}\left(u_{n}, v_{n}\right)\left(v_{n}-v\right)\right] d x .
\end{aligned}
\end{aligned}
$$

Then the fact $J^{\prime}\left(u_{n}, v_{n}\right) \rightarrow 0$ in $E^{*}$ implies $P_{n} \rightarrow 0$ as $n \rightarrow$ $\infty$. Similarly, the fact that $\left(u_{n}, v_{n}\right) \rightarrow(u, v)$ in $E$ implies that $Q_{n} \rightarrow 0$, where

$$
\begin{aligned}
Q_{n} & =\int_{\mathbb{R}^{N}}\left[|\nabla u|^{p-2} \nabla u \nabla\left(u_{n}-u\right)\right. \\
& \left.+m(x)|u|^{p-2} u\left(u_{n}-u\right)\right] d x \\
& +\int_{\mathbb{R}^{N}}\left[|\nabla v|^{q-2} \nabla v \nabla\left(v_{n}-v\right)\right. \\
& \left.+n(x)|v|^{q-2} v\left(v_{n}-u\right)\right] d x .
\end{aligned}
$$

Note that

$$
\begin{aligned}
& P_{n}-Q_{n}=T_{n}-\lambda \int_{\mathbb{R}^{N}}\left|u_{n}\right|^{p^{*}-2} u_{n}\left(u_{n}-u\right) d x \\
& -\mu \int_{\mathbb{R}^{N}}\left|v_{n}\right|^{q^{*}-2} v_{n}\left(v_{n}-v\right) d x-\frac{1}{\eta} \\
& \cdot \int_{\mathbb{R}^{N}}\left[G_{u}\left(u_{n}, v_{n}\right)\left(u_{n}-u\right)\right. \\
& \left.+G_{v}\left(u_{n}, v_{n}\right)\left(v_{n}-v\right)\right] d x,
\end{aligned}
$$

where

$$
\begin{aligned}
T_{n}= & \int_{\mathbb{R}^{N}}\left(\left|\nabla u_{n}\right|^{p-2} \nabla u_{n}-|\nabla u|^{p-2} \nabla u\right) \nabla\left(u_{n}-u\right) d x \\
& +\int_{\mathbb{R}^{N}} m(x)\left(\left|u_{n}\right|^{p-2} u_{n}-|u|^{p-2} u\right)\left(u_{n}-u\right) d x \\
& +\int_{\mathbb{R}^{N}}\left(\left|\nabla v_{n}\right|^{q-2} \nabla v_{n}-|\nabla v|^{q-2} \nabla v\right) \nabla\left(v_{n}-v\right) d x \\
& +\int_{\mathbb{R}^{N}} n(x)\left(\left|v_{n}\right|^{q-2} v_{n}-|v|^{q-2} v\right)\left(v_{n}-v\right) d x .
\end{aligned}
$$

If $T_{n} \rightarrow 0$, this implies $\left\|u_{n}-u\right\|_{X} \rightarrow 0$ and $\left\|v_{n}-v\right\|_{Y} \rightarrow 0$ according to the following lemma.

Lemma 6 (see [11]). There exists constant $C>0$ such that, for all $x, y \in \mathbb{R}^{N}$,

$$
\begin{aligned}
& \left(|x|^{p-2} x-|y|^{p-2} y, x-y\right) \\
& \geq C(|x|+|y|)^{p-2}|x-y|^{2}, \quad \text { if } 1<p<2, \\
& \left(|x|^{p-2} x-|y|^{p-2} y, x-y\right) \geq C|x-y|^{p}, \quad \text { if } p \geq 2,
\end{aligned}
$$

where $(\cdot, \cdot)$ denotes the usual scalar product in $\mathbb{R}^{N}$.

In the following, we will claim

$$
\begin{aligned}
\int_{\mathbb{R}^{N}} \lambda\left|u_{n}\right|^{p^{*}-2} u_{n}\left(u_{n}-u\right) d x & \longrightarrow 0, \\
\int_{\mathbb{R}^{N}} \mu\left|v_{n}\right|^{q^{*}-2} v_{n}\left(v_{n}-v\right) d x & \longrightarrow 0, \\
\text { as } n & \longrightarrow \infty .
\end{aligned}
$$

Based on concentration-compactness principle [12] and (12), it follows that

$$
\begin{gathered}
\left|\nabla u_{n}\right|^{p}-\mu \geq|\nabla u|^{p}+\sum_{j \in \mathscr{F}} \mu_{j} \delta_{x_{j}}, \\
\left|u_{n}\right|^{p^{*}}-v=\left|u_{n}\right|^{p^{*}}+\sum_{j \in \mathscr{\mathcal { F }}} v_{j} \delta_{x_{j}}, \\
\mu_{j} \geq S_{1} v_{j}^{p / p^{*}},
\end{gathered}
$$


where $\delta_{x_{j}}$ is the Dirac measure at $x_{j}$ and $\mu_{j}, v_{j}$ are nonnegative bounded measures. Analogously, we get $\left|\nabla v_{n}\right|^{q} \rightarrow \bar{\mu}$ and $\left|v_{n}\right|^{q^{*}} \rightarrow \bar{\nu}$. Concentration at $\infty$ of $\left\{u_{n}\right\}$ is defined by

$$
\begin{aligned}
& \mu_{\infty}=\lim _{R \rightarrow \infty} \limsup _{n \rightarrow \infty} \int_{|x|>R}\left|\nabla u_{n}\right|^{p} d x, \\
& \nu_{\infty}=\lim _{R \rightarrow \infty} \limsup _{n \rightarrow \infty} \int_{|x|>R}\left|u_{n}\right|^{p^{*}} d x .
\end{aligned}
$$

Analogously, the concentration at $\infty$ of $\left\{v_{n}\right\}$ is given by $\bar{\mu}_{\infty}$ and $\bar{\nu}_{\infty}$.

Step 1. For $j \in \mathcal{F}$, we define $\phi_{j} \in C_{0}^{\infty}\left(\mathbb{R}^{N}\right)$ as follows:

$$
\phi_{j}(x)= \begin{cases}1, & \text { if } x \in B\left(x_{j}, \epsilon\right), \\ 0, & \text { if } x \in R^{N} \backslash B\left(x_{j}, 2 \epsilon\right), \\ \left|\nabla \phi_{j}\right| \leq C, & \text { the other. }\end{cases}
$$

One can see that $\left\{u_{n} \phi_{j}\right\}$ is bounded in $X$; we obtain $\lim _{n \rightarrow \infty}\left\langle J^{\prime}\left(u_{n}, v_{n}\right),\left(u_{n} \phi_{j}, 0\right)\right\rangle=0$. Namely,

$$
\begin{aligned}
& -\lim _{n \rightarrow \infty} \int_{\mathbb{R}^{N}}\left|\nabla u_{n}\right|^{p-2} u_{n} \nabla u_{n} \nabla \phi_{j} d x \\
& \quad=\lim _{n \rightarrow \infty}\left[\int_{\mathbb{R}^{N}}\left(\left|\nabla u_{n}\right|^{p} \phi_{j}+m(x)\left|u_{n}\right|^{p} \phi_{j}\right) d x\right. \\
& \left.\quad-\lambda \int_{\mathbb{R}^{N}}\left|u_{n}\right|^{p^{*}} \phi_{j} d x-\frac{1}{\eta} \int_{\mathbb{R}^{N}} \frac{\partial G}{\partial u}\left(u_{n}, v_{n}\right) u_{n} \phi_{j} d x\right] .
\end{aligned}
$$

The fact that $u_{n} \rightarrow u$ produces $\int_{\mathbb{R}^{N}}(\partial G / \partial u)\left(u_{n}, v_{n}\right) u_{n} \phi_{j} d x \rightarrow$ $\int_{\mathbb{R}^{N}}(\partial G / \partial u)(u, v) u \phi_{j} d x$. In the light of Lions' principle, we acquire

$$
\begin{gathered}
\int_{\mathbb{R}^{N}}\left|\nabla u_{n}\right|^{p} \phi_{j} d x \longrightarrow \int_{\mathbb{R}^{N}} \phi_{j} d \mu, \\
\int_{\mathbb{R}^{N}}\left|u_{n}\right|^{p^{*}} \phi_{j} d x \longrightarrow \int_{\mathbb{R}^{N}} \phi_{j} d \nu .
\end{gathered}
$$

Moreover

$$
\begin{gathered}
\left.\lim _{\epsilon \rightarrow 0} \lim _{n \rightarrow \infty}\left|\int_{\mathbb{R}^{N}}\right| \nabla u_{n}\right|^{p-2} u_{n} \nabla u_{n} \nabla \phi_{j} d x \mid \\
\leq \lim _{\epsilon \rightarrow 0} \lim _{n \rightarrow \infty}\left(\int_{\mathbb{R}^{N}}\left|\nabla u_{n}\right|^{p} d x\right)^{(p-1) / p} \\
\cdot\left(\int_{\mathbb{R}^{N}}\left|u_{n} \nabla \phi_{j}\right|^{p} d x\right)^{1 / p} \leq M^{p-1} \\
\cdot \lim _{\epsilon \rightarrow 0} \lim _{n \rightarrow \infty}\left(\int_{\mathbb{R}^{N}}\left|u_{n} \nabla \phi_{j}\right|^{p} d x\right)^{1 / p} \leq M^{p-1} \\
\cdot \lim _{\epsilon \rightarrow 0} \lim _{n \rightarrow \infty}\left(\int_{\mathbb{B}_{2 \epsilon}\left(x_{j}\right)}\left|u_{n}\right|^{p^{*}} d x\right)^{1 / p^{*}}
\end{gathered}
$$

$$
\begin{gathered}
\cdot\left(\int_{\mathbb{B}_{2 \epsilon}\left(x_{j}\right)}\left|\nabla \phi_{j}\right|^{N} d x\right)^{1 / N} \leq C \\
\cdot \lim _{\epsilon \rightarrow 0}\left(\int_{\mathbb{B}_{2 \epsilon}\left(x_{j}\right)}|u|^{p^{*}} d x\right)^{1 / p^{*}}=0 .
\end{gathered}
$$

At the same time, we have

$$
\begin{aligned}
& \lim _{\epsilon \rightarrow 0} \lim _{n \rightarrow \infty} \int_{\mathbb{R}^{N}} \frac{\partial G}{\partial u}\left(u_{n}, v_{n}\right) u_{n} \phi_{j} d x \\
& \quad \leq \lim _{\epsilon \rightarrow 0} \lim _{n \rightarrow \infty} \int_{\mathbb{B}_{2 \epsilon}\left(x_{j}\right)} \beta(x)\left(\left|u_{n}\right|^{\eta}+\left|v_{n}\right|^{\eta}\right) \phi_{j} d x \\
& \quad \longrightarrow 0 \\
& \lim _{\epsilon \rightarrow 0} \lim _{n \rightarrow \infty} \int_{\mathbb{R}^{N}}\left|u_{n}\right|^{p} \phi_{j} d x \longrightarrow 0 .
\end{aligned}
$$

From the aforementioned equations, it follows that

$$
0=\lim _{\epsilon \rightarrow 0} \lim _{n \rightarrow \infty}\left\langle I^{\prime}\left(u_{n}, v_{n}\right),\left(u_{n} \phi_{j}, 0\right)\right\rangle=\mu_{j}-\lambda v_{j}
$$

combining with (36), we obtain

$$
\begin{gathered}
v_{j}=0 \\
\text { or } v_{j} \geq\left(\frac{S_{1}}{\lambda}\right)^{N / P} .
\end{gathered}
$$

Step 2. By choosing a suitable cut-off function $\varphi(x) \in$ $C^{\infty}\left(\mathbb{R}^{N},[0,1]\right)$ such that

$$
\varphi(x)= \begin{cases}0, & \text { if }|x| \leq 1 \\ 1, & \text { if }|x| \geq 2\end{cases}
$$

we set $\varphi_{R}(x)=\varphi(x / R)$; then $\left\{\varphi_{R} u_{n}\right\}$ is bounded in $X$ and $\lim _{R \rightarrow \infty}\left\langle J^{\prime}\left(u_{n}, v_{n}\right),\left(u_{n} \varphi_{R}, 0\right)\right\rangle=0$.

In a similar manner, we gain

$$
\begin{aligned}
& -\lim _{n \rightarrow \infty} \int_{\mathbb{R}^{N}}\left|\nabla u_{n}\right|^{p-2} u_{n} \nabla u_{n} \nabla \varphi_{R} d x \\
& \quad=\lim _{n \rightarrow \infty}\left[\int_{\mathbb{R}^{N}}\left(\left|\nabla u_{n}\right|^{p} \varphi_{R}+m(x)\left|u_{n}\right|^{p} \varphi_{R}\right) d x\right. \\
& -\lambda \int_{\mathbb{R}^{N}}\left|u_{n}\right|^{p^{*}} \varphi_{R} d x \\
& \left.-\frac{1}{\eta} \int_{\mathbb{R}^{N}} \frac{\partial G}{\partial u}\left(u_{n}, v_{n}\right) u_{n} \varphi_{R} d x\right] .
\end{aligned}
$$


Note that

$$
\begin{aligned}
& \left.\lim _{R \rightarrow \infty} \limsup _{n \rightarrow \infty}\left|\int_{\mathbb{R}^{N}}\right| \nabla u_{n}\right|^{p-2}\left|u_{n}\right|\left|\nabla u_{n}\right|\left|\nabla \varphi_{R}\right| d x \mid \\
& \leq \lim _{R \rightarrow \infty} \limsup _{n \rightarrow \infty}\left(\int_{\mathbb{R}^{N}}\left|\nabla u_{n}\right|^{p} d x\right)^{(p-1) / p} \\
& \cdot\left(\int_{\mathbb{R}^{N}}\left|u_{n} \nabla \varphi_{R}\right|^{p} d x\right)^{1 / p} \leq M^{p-1} \\
& \cdot \lim _{R \rightarrow \infty} \limsup _{n \rightarrow \infty}\left(\int_{\mathbb{R}^{N}}\left|u_{n} \nabla \varphi_{R}\right|^{p} d x\right)^{1 / p} \leq M^{p-1} \\
& \cdot \lim _{R \rightarrow \infty}\left[\left(\int_{R \leq|x| \leq 2 R}|u|^{p^{*}} d x\right)^{1 / p^{*}}\right. \\
& \left.\cdot\left(\int_{R \leq|x| \leq 2 R}\left|\nabla \varphi_{R}\right|^{N} d x\right)^{1 / N}\right] \leq C^{\prime} \\
& \cdot \lim _{R \rightarrow \infty}\left(\int_{R \leq|x| \leq 2 R}|u|^{p^{*}} d x\right)^{1 / p^{*}}=0,
\end{aligned}
$$

which deduces

$$
\begin{gathered}
v_{\infty}=0 \\
\text { or } v_{\infty} \geq\left(\frac{S_{1}}{\lambda}\right)^{N / P} .
\end{gathered}
$$

Next we will prove $v_{j} \geq\left(S_{p} / \lambda\right)^{N / P}$ and $v_{\infty} \geq\left(S_{p} / \lambda\right)^{N / P}$ are impossible.

Case 1. If we fix $\eta \in\left(q, p^{*}\right)$, there exists $j_{0}$ such that $\nu_{j_{0}} \geq$ $\left(S_{p} / \lambda\right)^{N / P}$; then one can get

$$
\begin{aligned}
J\left(u_{n}, v_{n}\right)-J^{\prime}\left(u_{n}, v_{n}\right)\left(\frac{u_{n}}{p}, \frac{v_{n}}{q}\right) \\
=\left(\frac{1}{p}-\frac{1}{p^{*}}\right) \int_{\mathbb{R}^{N}} \lambda\left|u_{n}\right|^{P^{*}} d x \\
\quad+\left(\frac{1}{q}-\frac{1}{q^{*}}\right) \int_{\mathbb{R}^{N}} \mu\left|v_{n}\right|^{q^{*}} d x \\
\quad+\frac{1}{\eta} \int_{\mathbb{R}^{N}}\left[\frac{1}{p} G_{u} u_{n}+\frac{1}{q} G_{v} v_{n}+G\left(u_{n}, v_{n}\right)\right] d x \\
\geq \frac{\lambda}{N} \int_{\mathbb{R}^{N}}\left|u_{n}\right|^{P^{*}} d x+\frac{\mu}{N} \int_{\mathbb{R}^{N}}\left|v_{n}\right|^{q^{*}} d x .
\end{aligned}
$$

$$
\geq \frac{\lambda}{N}\left(\frac{S_{1}}{\lambda}\right)^{N / P}+\frac{\mu}{N}\left(\frac{S_{2}}{\mu}\right)^{N / q},
$$

which gives rise to a contradiction, so $v_{j}=0$ for all $j \in \mathscr{J}$. With the similar argument, we can get $\mu_{\infty}=\nu_{\infty}=0$.

Case 2. If we fix $\eta \in(1, p)$, one can see

$$
\begin{aligned}
0> & c=\lim _{n \rightarrow \infty}\left[J\left(u_{n}, v_{n}\right)-J^{\prime}\left(u_{n}, v_{n}\right)\left(\frac{u_{n}}{p^{*}}, \frac{v_{n}}{q^{*}}\right)\right] \\
= & \left(\frac{1}{p}-\frac{1}{p^{*}}\right)\left\|u_{n}\right\|_{X}^{p}+\left(\frac{1}{q}-\frac{1}{q^{*}}\right)\left\|v_{n}\right\|_{Y}^{q} \\
& -\left(\frac{1}{\eta}-\frac{1}{q^{*}}\right) \int_{\mathbb{R}^{N}} G\left(u_{n}, v_{n}\right) d x \geq \frac{1}{N} \\
& \cdot \min \left\{\left\|\left(u_{n}, v_{n}\right)\right\|_{E}^{p},\left\|\left(u_{n}, v_{n}\right)\right\|_{E}^{q}\right\}-\left(\frac{1}{\eta}-\frac{1}{q^{*}}\right) \\
& \cdot \int_{\mathbb{R}^{N}} \beta(x)\left(\left|u_{n}\right|^{\eta}+\left|v_{n}\right|^{\eta}\right) d x \geq \frac{1}{N} \\
& \cdot \min \left\{\left\|\left(u_{n}, v_{n}\right)\right\|_{E}^{p},\left\|\left(u_{n}, v_{n}\right)\right\|_{E}^{q}\right\}-\left(\frac{1}{\eta}-\frac{1}{q^{*}}\right) \\
& \cdot\left(\|\beta(x)\|_{9} S_{1}^{-\eta / p}\left\|u_{n}\right\|_{X}^{\eta}+\|\beta(x)\|_{\gamma} S_{2}^{-\eta / q}\left\|v_{n}\right\|_{Y}^{\eta}\right) \\
& \geq \frac{1}{N} \min \left\{\left\|\left(u_{n}, v_{n}\right)\right\|_{E}^{p},\left\|\left(u_{n}, v_{n}\right)\right\|_{E}^{q}\right\}-\left(\frac{1}{\eta}-\frac{1}{q^{*}}\right) \\
& \cdot \max \left\{\|\beta(x)\|_{9} S_{1}^{-\eta / p},\|\beta(x)\|_{\gamma} S_{2}^{-\eta / q}\right\}\left\|\left(u_{n}, v_{n}\right)\right\|_{E}^{\eta} ;
\end{aligned}
$$

if $\min \left\{\left\|\left(u_{n}, v_{n}\right)\right\|_{E}^{p},\left\|\left(u_{n}, v_{n}\right)\right\|_{E}^{q}\right\}=\left\|\left(u_{n}, v_{n}\right)\right\|_{E}^{p}$ we can obtain

$$
\begin{aligned}
& \left\|\left(u_{n}, v_{n}\right)\right\|_{E} \leq\left(N\left(\frac{1}{\eta}-\frac{1}{q^{*}}\right)\right. \\
& \left.\cdot \max \left\{\|\beta(x)\|_{\vartheta} S_{1}^{-\eta / p},\|\beta(x)\|_{\gamma} S_{2}^{-\eta / q}\right\}\right)^{1 /(p-\eta)} ;
\end{aligned}
$$

otherwise,

$$
\begin{aligned}
& \left\|\left(u_{n}, v_{n}\right)\right\|_{E} \leq\left(N\left(\frac{1}{\eta}-\frac{1}{q^{*}}\right)\right. \\
& \left.\cdot \max \left\{\|\beta(x)\|_{9} S_{1}^{-\eta / p},\|\beta(x)\|_{\gamma} S_{2}^{-\eta / q}\right\}\right)^{1 /(q-\eta)} ;
\end{aligned}
$$

moreover,

$$
\begin{gathered}
0>c=\lim _{R \rightarrow \infty} \lim _{n \rightarrow \infty}\left[J\left(u_{n}, v_{n}\right)\right. \\
\left.-J^{\prime}\left(u_{n}, v_{n}\right)\left(\frac{u_{n}}{p^{*}} \varphi_{R}, \frac{v_{n}}{q^{*}} \varphi_{R}\right)\right] \geq\left(\frac{1}{p}-\frac{1}{p^{*}}\right) \mu_{\infty} \\
+\left(\frac{1}{q}-\frac{1}{q^{*}}\right) \bar{\mu}_{\infty}-\left(\frac{1}{\eta}-\frac{1}{q^{*}}\right) \max \left\{\|\beta(x)\|_{9} S_{1}^{-\eta / p},\right. \\
\left.\|\beta(x)\|_{\gamma} S_{2}^{-\eta / q}\right\}\left\|\left(u_{n}, v_{n}\right)\right\|_{E}^{\eta}=\frac{1}{N}\left(\mu_{\infty}+\overline{\mu_{\infty}}\right)-\left(\left(\frac{1}{\eta}\right.\right. \\
\left.\left.-\frac{1}{q^{*}}\right) \max \left\{\|\beta(x)\|_{9} S_{1}^{-\eta / p},\|\beta(x)\|_{\gamma} S_{2}^{-\eta / q}\right\}\right)^{p /(p-\eta)}
\end{gathered}
$$




$$
\begin{aligned}
& \cdot N^{\eta /(p-\eta)} \geq \frac{1}{N}\left(\left(\frac{S_{1}}{\lambda}\right)^{N / p}+\left(\frac{S_{2}}{\mu}\right)^{N / q}\right)-\left(\left(\frac{1}{\eta}\right.\right. \\
& \left.\left.-\frac{1}{q^{*}}\right) \max \left\{\|\beta(x)\|_{\vartheta} S_{1}^{-\eta / p},\|\beta(x)\|_{\gamma} S_{2}^{-\eta / q}\right\}\right)^{p /(p-\eta)} \\
& \cdot N^{\eta /(p-\eta)} .
\end{aligned}
$$

We can easily get that there exists $\Lambda_{0}>0$ being small such that when $0<\lambda, \mu<\Lambda_{0}$, the right side is positive, which gives rise to a contradiction.

Up to now, we have shown that

$$
\begin{aligned}
& \lim _{n \rightarrow \infty} \int_{\mathbb{R}^{N}}\left|u_{n}\right|^{p^{*}}=\int_{\mathbb{R}^{N}}|u|^{p^{*}} \\
& \lim _{n \rightarrow \infty} \int_{\mathbb{R}^{N}}\left|v_{n}\right|^{q^{*}}=\int_{\mathbb{R}^{N}}|v|^{q^{*}} .
\end{aligned}
$$

In view of (12) and the Brezis-Lieb Lemma [13], we acquire

$$
\begin{aligned}
& \lim _{n \rightarrow \infty} \int_{\mathbb{R}^{N}}\left|u_{n}-u\right|^{p^{*}} d x=0, \\
& \left.\lim _{n \rightarrow \infty} \int_{\mathbb{R}^{N}}\left|v_{n}-v\right|\right|^{p^{*}} d x=0 .
\end{aligned}
$$

As a result of the above, it yields $\left\|\left(u_{n}-u, v_{n}-v\right)\right\|_{E} \rightarrow 0$, which implies the functional of (1) fulfils $(P S)_{c}$ condition.

\section{Proof of Theorem 1}

The purpose of this section is to give proof of Theorem 1 which mainly relies on mountain pass lemma as follows.

Lemma 7 (see [14]). Let $X$ be a real Banach space, $J \in$ $C^{1}(E, R), J(0)=0$. If

$\left(\mathscr{P}_{1}\right)$ there exist constants $r, b>0$ such that $J(u) \geq b$ for all $u \in X$ with $\|u\|_{X}=r$,

$\left(\mathscr{P}_{2}\right)$ there is $e,\|e\|_{X}>r$, such that $J(e) \leq 0$,

$\left(\mathscr{P}_{3}\right)$ the functional $J(u)$ satisfies $P S$ condition: every sequence $\left\{u_{n}\right\} \subset X$, such that $J\left(u_{n}\right) \rightarrow c$ for some $c$, and $J^{\prime}\left(u_{n}\right) \rightarrow 0$ in $X^{*}$ as $n \rightarrow \infty$, is relatively compact in $X$,

then J possesses a critical value $c \geq b$ which can be characterized as

$$
c=\inf _{g \in \Gamma} \max _{t \in[0,1]} J(g(t))
$$

where

$$
\Gamma=\{g \in C([0,1], X) \mid g(0)=0, g(1)=e\} .
$$

In the following, we want to show that

$$
c<c^{*}=\min \left\{\frac{\lambda}{N}\left(\frac{S_{1}}{\lambda}\right)^{N / P}, \frac{\mu}{N}\left(\frac{S_{2}}{\mu}\right)^{N / q}\right\} .
$$

We suppose $c^{*}=(\lambda / N)\left(S_{1} / \lambda\right)^{N / P}$ and we can get the other case with similar arguments. Let $\omega=u /\|u\|_{p^{*}} \in X \backslash\{0\}$; then we get the following lemma.
Lemma 8. Under hypotheses $\left(\mathscr{H}_{1}\right),\left(\mathscr{H}_{2}\right)$, and $\left(\mathscr{H}_{3}\right)$ with $\eta \epsilon$ $\left(q, p^{*}\right)$, there exists $\Lambda_{0}>0$ such that if $0<\lambda<\Lambda_{0}$, we have $0<\sup _{t \geq 0} J(t \omega, 0)<c^{*}$.

Proof. For

$$
\begin{aligned}
J(t \omega, 0)= & \frac{t^{p}}{p}\|\omega\|_{X}^{p}-\frac{t^{p^{*}}}{p^{*}} \int_{\mathbb{R}^{N}} \lambda|\omega|^{p^{*}} d x \\
& -\frac{1}{\eta} \int_{\mathbb{R}^{N}} G(t \omega, 0) d x \\
= & \frac{t^{p}}{p}\|\omega\|_{X}^{p}-\frac{t^{p^{*}}}{p^{*}} \int_{\mathbb{R}^{N}} \lambda|\omega|^{p^{*}} d x \\
& -\frac{t^{\eta}}{\eta} \int_{\mathbb{R}^{N}} G(\omega, 0) d x
\end{aligned}
$$

with $p<\eta<p^{*}$, we can easily get $J(t \omega, 0) \rightarrow-\infty$ as $t \rightarrow \infty$, $J\left(t_{0} \omega, 0\right)<0$ with $t_{0}$ large enough, and $J(t \omega, 0) \geq r>0$ with $t$ sufficiently small. So $0<\sup _{t \geq 0} J(t \omega, 0)=J\left(t_{\lambda} \omega, 0\right)$ for some $t_{\lambda}>0$; namely,

$$
\begin{aligned}
J\left(t_{\lambda} \omega, 0\right)= & \frac{t_{\lambda}^{p}}{p}\|\omega\|_{X}^{p}-\frac{t_{\lambda}^{p^{*}}}{p^{*}} \int_{\mathbb{R}^{N}} \lambda|\omega|^{p^{*}} d x \\
& -\frac{t_{\lambda}^{\eta}}{\eta} \int_{\mathbb{R}^{N}} G(\omega, 0) d x .
\end{aligned}
$$

We can have $J^{\prime}\left(t_{\lambda} \omega, 0\right)\left(t_{\lambda} \omega, 0\right)=0$; that is,

$$
\begin{aligned}
t_{\lambda}^{p}\|\omega\|_{X}^{p}-t_{\lambda}^{p^{*}} \int_{\mathbb{R}^{N}} \lambda|\omega|^{p^{*}} d x \\
\quad-\frac{1}{\eta} \int_{\mathbb{R}^{N}} G_{u}\left(t_{\lambda} \omega, 0\right)\left(t_{\lambda} \omega, 0\right) d x=0 .
\end{aligned}
$$

By direct computation $t_{\lambda}^{p}\|\omega\|_{X}^{p} \geq t_{\lambda}^{p^{*}} \int_{\mathbb{R}^{N}} \lambda|\omega|^{p^{*}} d x$, we can get $t_{\lambda} \leq\left(S_{1} / \lambda\right)^{(N-p) / p^{2}}$.

Meanwhile

$$
\begin{aligned}
c & \leq \max _{t \geq 0} J(t \omega, 0)=\max _{t \geq 0}\left\{\frac{t_{\lambda}^{p}}{p}\|\omega\|_{X}^{p}\right. \\
& \left.-\frac{t_{\lambda}^{p^{*}}}{p^{*}} \int_{\mathbb{R}^{N}} \lambda|\omega|^{p^{*}} d x-\frac{t_{\lambda}^{\eta}}{\eta} \int_{\mathbb{R}^{N}} G(\omega, 0) d x\right\} \\
& \leq \max _{t \geq 0}\left\{\frac{t_{\lambda}^{p}}{p}\|\omega\|_{X}^{p}-\frac{t_{\lambda}^{p^{*}}}{p^{*}} \int_{\mathbb{R}^{N}} \lambda|\omega|^{p^{*}} d x\right\} .
\end{aligned}
$$

Denote $F(t)=\left(t^{p} / p\right)\|\omega\|_{X}^{p}-\left(t^{p^{*}} / p^{*}\right) \int_{\mathbb{R}^{N}} \lambda|\omega|^{p^{*}} d x, t>0$; it can achieve its maximum at

$$
\begin{aligned}
t & =\left(\frac{\|\omega\|_{X}^{p}}{\int_{\mathbb{R}^{N}} \lambda|\omega|^{p^{*}} d x}\right)^{(N-p) / p^{2}} \\
& =\left(\frac{S_{1}}{\lambda}\right)^{(N-p) / p^{2}}
\end{aligned}
$$




$$
\begin{aligned}
\sup _{t>0} F(t)= & F\left(\frac{S_{1}}{\lambda}\right) \\
= & \frac{\|\omega\|_{X}^{p}}{p}\left(\left(\frac{S_{1}}{\lambda}\right)^{(N-p) / p^{2}}\right)^{p} \\
& -\frac{\int_{\mathbb{R}^{N}} \lambda|\omega|^{p^{*}} d x}{p^{*}}\left(\left(\frac{S_{1}}{\lambda}\right)^{(N-p) / p^{2}}\right)^{p^{*}} \\
= & \frac{\|\omega\|_{X}^{p}}{p}\left(\frac{S_{1}}{\lambda}\right)^{(N-p) / p} \\
& -\frac{\int_{\mathbb{R}^{N}} \lambda|\omega|^{p^{*}} d x}{p^{*}}\left(\frac{S_{1}}{\lambda}\right)^{N / p} \\
= & \frac{\lambda}{N}\left(\frac{S_{1}}{\lambda}\right)^{N / P}=c^{*}
\end{aligned}
$$

thus we get $0<\sup _{t \geq 0} J(t \omega)<c^{*}$.

Proof of Theorem 1. Obviously, functional $J(u, v)$ satisfies conditions $\left(\mathscr{P}_{1}\right)-\left(\mathscr{P}_{3}\right)$ of the Lemma 7 we can define $c=$ $\inf _{g \in \Gamma} \max _{t \in[0,1]} J(g(t))$, where $\Gamma$ denotes the class of all continuous paths $g(t)$ joining the origin to $t_{0} \omega$. Thus, mountain pass lemma can be applied to conclude that $J(u, v)$ has a critical point with corresponding critical value $c$. So, system (1) has at least one nontrivial solution.

\section{Proof of Theorem 2}

In this section, we want to apply genus theory to get the main conclusion of Theorem 2. Let $X$ be a Banach space and $\Sigma$ be the class of closed and symmetric subsets with respect to the origin of $X-\{0\}$. For $Z \in \Sigma$, genus $\gamma(Z)$ is given by

$$
\begin{aligned}
\gamma(Z) & =\min \left\{k \in \mathbb{N}: \exists \varphi \in C\left(Z, \mathbb{R}^{k} \backslash\{0\}\right), \varphi(x)\right. \\
& =-\varphi(-x)\} .
\end{aligned}
$$

Since $\beta(x) \in L^{\gamma}\left(\mathbb{R}^{N}\right)$ with $\gamma=q^{*} /\left(q^{*}-\eta\right), \eta \in(1, p)$, thus we can have

$$
\begin{aligned}
& \int_{\mathbb{R}^{N}} G(u, v) d x \leq \int_{\mathbb{R}^{N}} \beta(x)\left(|u|^{\eta}+|v|^{\eta}\right) d x \\
& \quad \leq\|\beta(x)\|_{9}\|u\|_{p^{*}}^{\eta}+\|\beta(x)\|_{\gamma}\|v\|_{q^{*}}^{\eta} \\
& \quad \leq\|\beta(x)\|_{9} S_{1}^{-\eta / p}\|u\|_{X}^{\eta}+\|\beta(x)\|_{\gamma} S_{2}^{-\eta / q}\|v\|_{Y}^{\eta} \\
& \quad \leq \max \left\{\|\beta(x)\|_{9} S_{1}^{-\eta / p},\|\beta(x)\|_{\gamma} S_{2}^{-\eta / q}\right\}\|(u, v)\|_{E}^{\eta},
\end{aligned}
$$

where $\vartheta=p^{*} /\left(p^{*}-\eta\right) \geq \gamma$.

Moreover,

$$
\begin{aligned}
& \int_{\mathbb{R}^{N}}\left(\frac{\lambda}{p^{*}}|u|^{p^{*}}+\frac{\mu}{q^{*}}|v|^{q^{*}}\right) d x \leq \frac{\lambda}{p^{*}} S_{1}^{-p^{*} / p}\|u\|_{X}^{p^{*}} \\
& +\frac{\mu}{q^{*}} S_{2}^{-q^{*} / q}\|v\|_{Y}^{q^{*}} \leq \max \left\{\frac{\lambda}{p^{*}} S_{1}^{-p^{*} / p}, \frac{\mu}{q^{*}} S_{2}^{-q^{*} / q}\right\} \\
& \cdot \max \left\{\|(u, v)\|_{E}^{p^{*}},\|(u, v)\|_{E}^{q^{*}}\right\} .
\end{aligned}
$$

The corresponding functional $J(u, v)$ of (1) turns into

$$
\begin{aligned}
& J(u, v)=\frac{1}{p}\|u\|_{X}^{p}+\frac{1}{q}\|v\|_{Y}^{q}-\frac{\lambda}{p^{*}} \int_{\mathbb{R}^{N}}|u|^{p^{*}} d x-\frac{\mu}{q^{*}} \\
& \cdot \int_{\mathbb{R}^{N}}|v|^{q^{*}} d x-\frac{1}{\eta} \int_{\mathbb{R}^{N}} G(u, v) d x \geq \frac{1}{q} \\
& \cdot \min \left\{\|(u, v)\|_{E}^{p},\|(u, v)\|_{E}^{q}\right\} \\
& -\max \left\{\frac{\lambda}{p^{*}} S_{1}^{-p^{*} / p}, \frac{\mu}{q^{*}} S_{2}^{-q^{*} / q}\right\} \\
& \cdot \max \left\{\|(u, v)\|_{E}^{p^{*}},\|(u, v)\|_{E}^{q^{*}}\right\} \\
& -\frac{1}{\eta} \max \left\{\|\beta(x)\|_{9} S_{1}^{-\eta / p},\|\beta(x)\|_{\gamma} S_{2}^{-\eta / q}\right\}\|(u, v)\|_{E}^{\eta} \\
& \quad=\frac{1}{q} \min \left\{\|(u, v)\|_{E}^{p},\|(u, v)\|_{E}^{q}\right\}-b_{1} \\
& \quad \cdot \max \left\{\|(u, v)\|_{E}^{p^{*}},\|(u, v)\|_{E}^{q^{*}}\right\}-b_{2}\|(u, v)\|_{E}^{\eta},
\end{aligned}
$$

where $b_{1}=\max \left\{\left(\lambda / p^{*}\right) S_{1}^{-p^{*} / p},\left(\mu / q^{*}\right) S_{2}^{-q^{*} / q}\right\}$ and $b_{2}=(1 /$ $\eta) \max \left\{\|\beta(x)\|_{\vartheta} S_{1}^{-\eta / p},\|\beta(x)\|_{\gamma} S_{2}^{-\eta / q}\right\}$.

Define

$$
h(x)=\frac{1}{q} \min \left\{x^{p}, x^{q}\right\}-b_{1} \max \left\{x^{p^{*}}, x^{q^{*}}\right\}-b_{2} x^{\eta}
$$

we can observe that there exists $\Lambda_{1}>0$ being small such that if $0<\lambda, \mu<\Lambda_{1}$, there exists $0<x_{0}<x_{1}$ such that $h(x)$ becomes $-, 0,+, 0,-$ if $x \in\left(0, x_{0}\right), x_{0},\left(x_{0}, x_{1}\right), x_{1},\left(x_{1},+\infty\right)$, respectively.

We set up the truncated functional [15]

$$
\begin{aligned}
F(u, v)= & \frac{1}{p}\|u\|_{X}^{p}+\frac{1}{q}\|v\|_{Y}^{q} \\
& -\psi(u, v)\left(\int_{\mathbb{R}^{N}} \frac{\lambda}{p^{*}}|u|^{p^{*}}+\frac{\mu}{q^{*}}|v|^{q^{*}} d x\right) \\
& -\frac{1}{\eta} \int_{\mathbb{R}^{N}} G(u, v) d x,
\end{aligned}
$$

where $\psi(u, v)=\psi(z)=\omega\left(\|z\|_{E}\right)$ and $\omega: \mathbb{R}^{+} \rightarrow[0,1]$ is a nonincreasing $C^{\infty}$ function such that $\omega(x)=1$ for $x \leq x_{0}$ and $\omega(x)=0$ for $x \geq x_{1}$. Obviously, $F(u, v)$ is even and $F(u, v)$ satisfies $(P S)_{c}$ with $c<0$.

Lemma 9. Assume $\left(\mathscr{H}_{1}\right),\left(\mathscr{H}_{2}\right)$, and $\left(\mathscr{H}_{4}\right)$ with $\eta \in(1, p)$ hold. Given $n \in \mathbb{N}$, there exists $\epsilon=\epsilon(n)<0$ such that

$$
\gamma(\{(u, v) \in E: F(u, v) \leq \epsilon\}) \geq n .
$$


Proof. Fix $n$, and suppose $E_{n}$ is $n$-dimensional subspace of $E$. We can take $\left(u_{n}, v_{n}\right) \in E_{n}$, with $\left\|\left(u_{n}, v_{n}\right)\right\|_{E}=1$. Thus, for $0<\rho<x_{0}$, we have

$$
\begin{aligned}
& F\left(\rho u_{n}, \rho v_{n}\right)=J\left(\rho u_{n}, \rho v_{n}\right) \\
& \leq \frac{1}{p} \rho^{p} \\
& \quad-\min \left\{\frac{\lambda}{p^{*}}, \frac{\mu}{q^{*}}\right\} \rho^{q^{*}}\left(\int_{\mathbb{R}^{N}}|u|^{p^{*}}+|v|^{q^{*}} d x\right) \\
& \quad-\frac{1}{\eta} \rho^{\eta} \int_{\mathbb{R}^{N}} G(u, v) d x ;
\end{aligned}
$$

we can choose $\epsilon<0$ and $\varsigma<x_{0}$ small enough such that $F(\varsigma u, \varsigma v) \leq \epsilon$. Let $S_{\varsigma}=\left\{(u, v) \in E,\|(u, v)\|_{E}=\varsigma\right\}$; then, $S_{\varsigma} \cap E_{n} \subset \gamma(\{u \in E: F(u, v) \leq \epsilon\})$. Hence, $\gamma(\{(u, v) \in E$ : $F(u, v) \leq \epsilon\}) \geq \gamma\left(S_{\varsigma} \cap E_{n}\right)=n$.

Lemma 10. Denote $\Sigma_{k}=\{Z \in \Sigma: \gamma(Z) \geq k\}$ and set

$$
\begin{aligned}
& c_{k}=\inf _{Z \in \Sigma_{k}} \sup _{(u, v) \in Z} F(u, v), \\
& B_{c}=\left\{(u, v) \in E: F^{\prime}(u, v)=0, F(u, v)=c\right\} ;
\end{aligned}
$$

then, if $c=c_{k}=c_{k+1}=\cdots=c_{k+r}, \gamma\left(B_{c}\right) \geq r+1$

Proof. For convenience, we denote $F^{c}=\{\{(u, v) \in E$ : $F(u, v) \leq c\}\}$. Suppose $c=c_{k}=c_{k+1}=\cdots=c_{k+r}$; we observe that $c<0$ and $B_{c}$ is a compact set. If $\gamma\left(B_{c}\right) \leq r$, there exists a symmetric and closed set $W_{1}, B_{c} \subset W_{1}$ such that $\gamma\left(W_{1}\right) \leq r$. According to deformation lemma, one can get an odd homeomorphism $\varrho: E \rightarrow E$ such that

$$
\varrho\left(F^{c+\delta} \backslash W_{1}\right) \subset F^{c-\delta}, \quad f \text { or } \delta>0 .
$$

Based on the definition $c=c_{k+r}$, there exists $W_{2} \in \Sigma_{k+r}$ such that $\sup _{(u, v) \in W_{2}} F(u, v)<c+\delta$ and

$$
\varrho\left(W_{2} \backslash W_{1}\right) \subset \rho\left(F^{c+\delta} \backslash W_{1}\right) \subset F^{c-\delta} .
$$

Hence we gain

$$
\begin{aligned}
\gamma\left(\overline{W_{2} \backslash W_{1}}\right) & \geq \gamma\left(W_{2}\right)-\gamma\left(W_{1}\right) \geq k, \\
\gamma\left(\varrho\left(\overline{W_{2} \backslash W_{1}}\right)\right) & \geq \gamma\left(\overline{W_{2} \backslash W_{1}}\right) \geq k .
\end{aligned}
$$

Therefore, $\varrho\left(\overline{W_{2} \backslash W_{1}}\right) \in \Sigma_{k}$ and it is in contradiction to (76). Lemma 10 and Kajikiya's [16] symmetric mountain pass lemma give the proof of Theorem 2 .

\section{Conflicts of Interest}

The authors declare that they have no conflicts of interest.

\section{Acknowledgments}

This work is supported by National Natural Science Foundation of China (11701252) and Project of Shandong Province Higher Educational Science and Technology Program (J16LI03).

\section{References}

[1] H. Brezis and L. Nirenberg, "Positive solutions of nonlinear elliptic equations involving critical Sobolev exponents," Communications on Pure and Applied Mathematics, vol. 36, no. 4, pp. 437-477, 1983.

[2] T.-F. Wu, "A semilinear elliptic problem involving nonlinear boundary condition and sign-changing potential," Electronic Journal of Differential Equations, vol. 131, pp. 1-15, 2006.

[3] T.-F. Wu, "Multiplicity of positive solution of p-Laplacian problems with Sign-Changing weight functions," Journal of Mathematical Analysis and Applications, vol. 12, pp. 557-563, 2007.

[4] T.-F. Wu, "Multiple positive solutions for semilinear elliptic systems with nonlinear boundary condition," Applied Mathematics and Computation, vol. 189, no. 2, pp. 1712-1722, 2007.

[5] J. Garcia-Azorero, I. Peral, and J. D. Rossi, "A convex-concave problem with a nonlinear boundary condition," Journal of Differential Equations, vol. 198, no. 1, pp. 91-128, 2004.

[6] H. H. Yin and Z. D. Yang, "Multiplicity results for a class of concave-convex elliptic systems involving sign-changing weight functions," Annales Polonici Mathematici, vol. 102, no. 1, pp. 5171, 2011.

[7] D. Ali and S. Tas, "Quasilinear elliptic systems with critical Sobolev exponents in RN," Nonlinear Analysis, vol. 66, no. 7, pp. 1485-1497, 2007.

[8] H. Yan and D. S. Kang, "On the singular elliptic systems involving multiple critical Sobolev exponents," Nonlinear Analysis, vol. 74, pp. 300-412, 2011.

[9] E. S. Noussair and C. A. Swanson, "Multiple finite energy states for critical potential systems," Nonlinear Analysis, vol. 26, no. 2, pp. 217-240, 1996.

[10] T.-S. Hsu, "Multiple positive solutions for a critical quasilinear elliptic system with concave-convex nonlinearities," Nonlinear Analysis, vol. 71, no. 7-8, pp. 2688-2698, 2009.

[11] G. Dinca and P. Jebelean, "Some existence results for a class of nonlinear equations involving a duality mapping," Nonlinear Analysis, vol. 46, no. 3, pp. 347-363, 2001.

[12] P.-L. Lions, "The concentration-compactness principle in the calculus of variations. The locally compact case, Parts 1 and 2," Annales de l'Institut Henri Poincaré C, Analyse Non Linéaire, vol. 1, pp. 109-145, 223-283, 1984.

[13] H. Brezis and E. H. Lieb, "A relation between pointwise convergence of functions and convergence of functionals," Proceedings of the American Mathematical Society, vol. 88, no. 3, pp. 486490, 1983.

[14] P. H. Rabinowitz, Minimax Methods in Critical Point Theory with Applications to Differential Equations, vol. 65 of CBMS Regional Conference Series in Mathematics, American Mathematical Society, Providence, RI, USA, 1986.

[15] F. Bernis, J. Garcia-Azorero, and I. Peral, "Existence and multiplicity of nontrivial solutions in semilinear critical problems of fourth order," Advances in Differential Equations, vol. 1, no. 2, pp. 219-240, 1996.

[16] R. Kajikiya, "A critical point theorem related to the symmetric mountain pass lemma and its applications to elliptic equations," Journal of Functional Analysis, vol. 225, no. 2, pp. 352-370, 2005. 


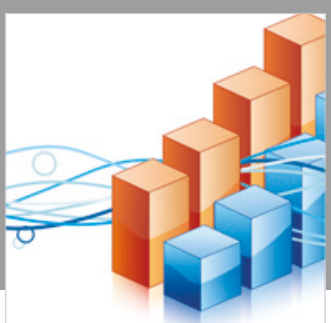

Advances in

Operations Research

\section{-n-m}
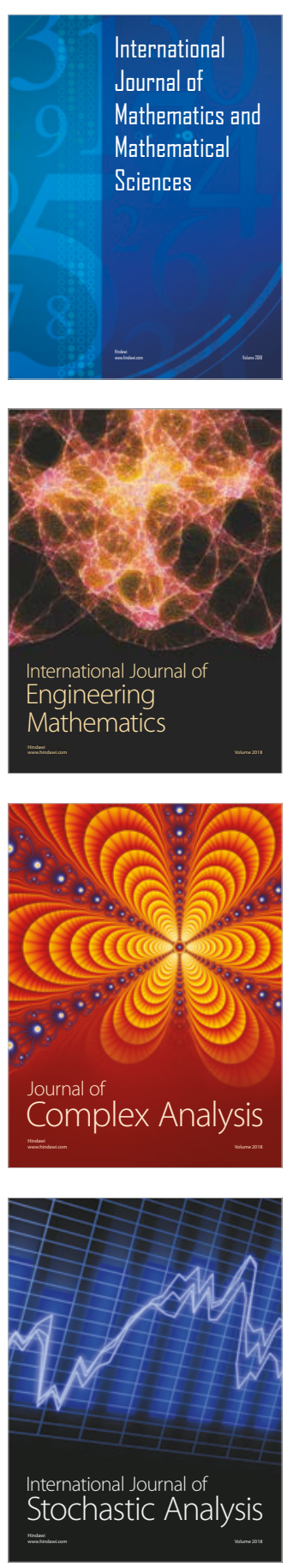
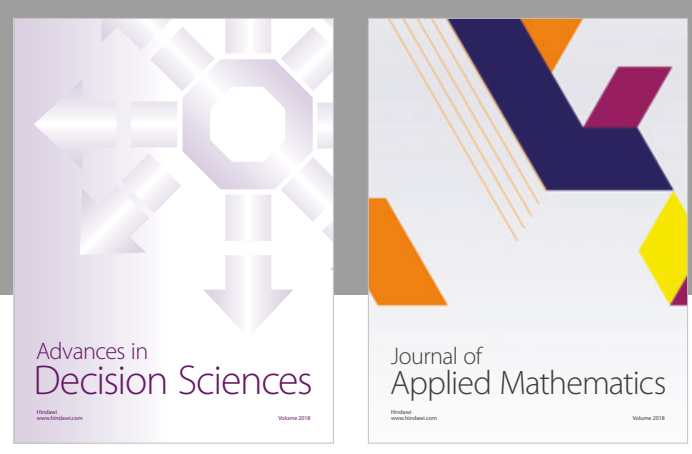

Journal of

Applied Mathematics
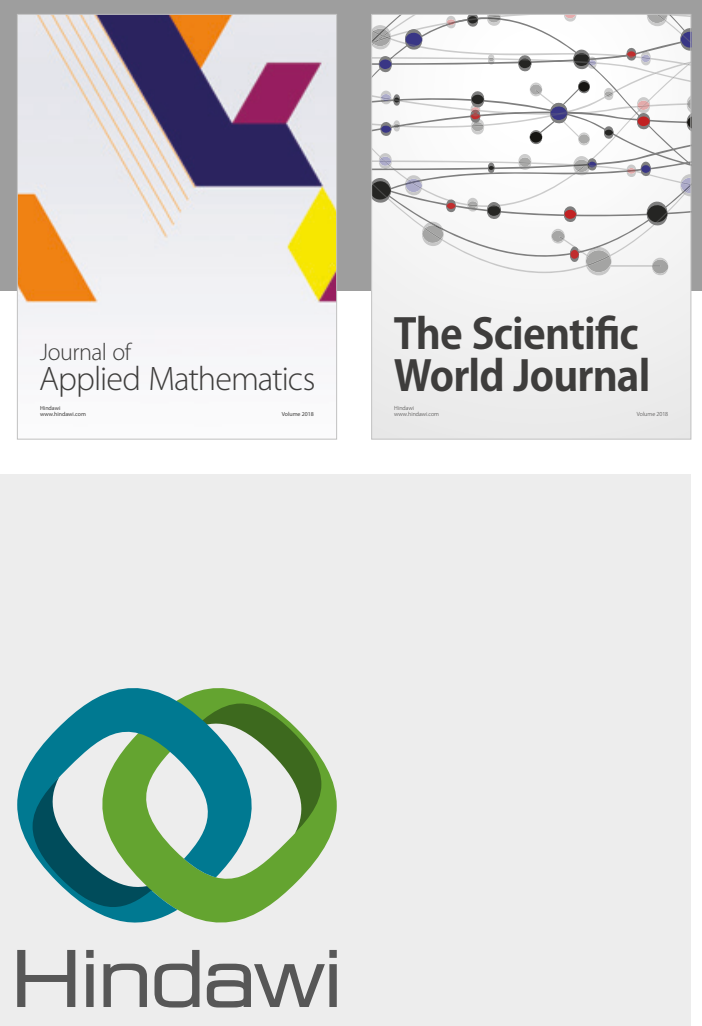

Submit your manuscripts at

www.hindawi.com

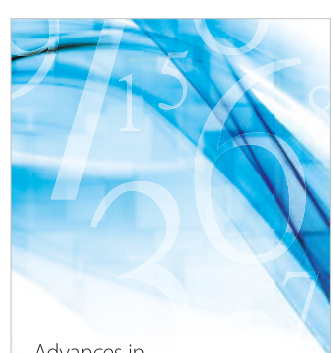

Advances in
Numerical Analysis
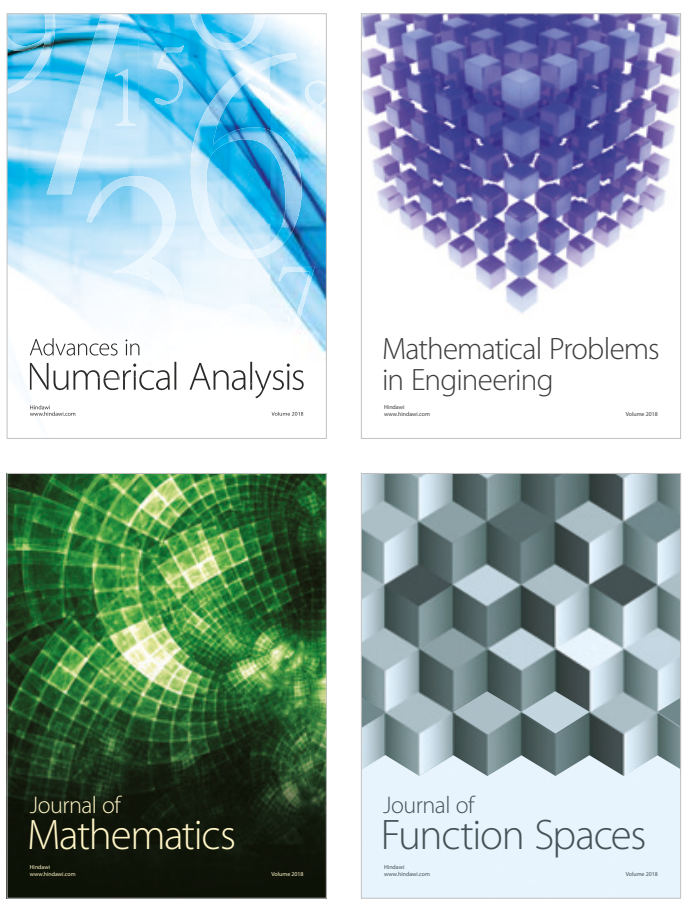

Mathematical Problems in Engineering

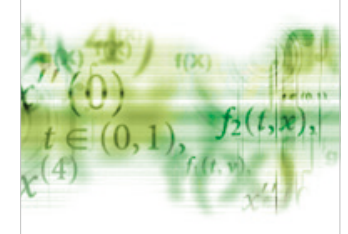

International Journal of

Differential Equations

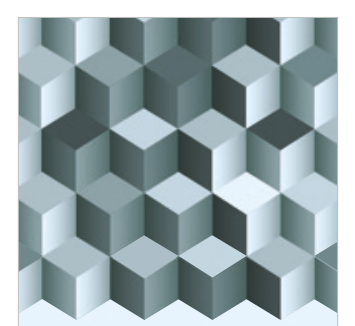

Journal of

Function Spaces

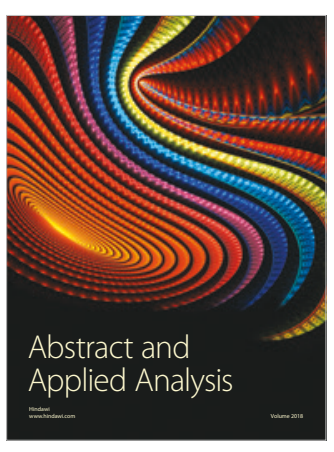

The Scientific

World Journal

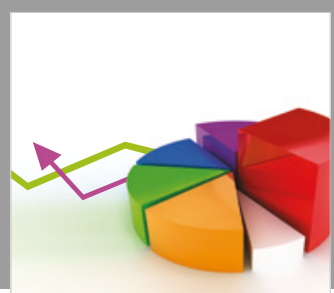

Journal of

Probability and Statistics
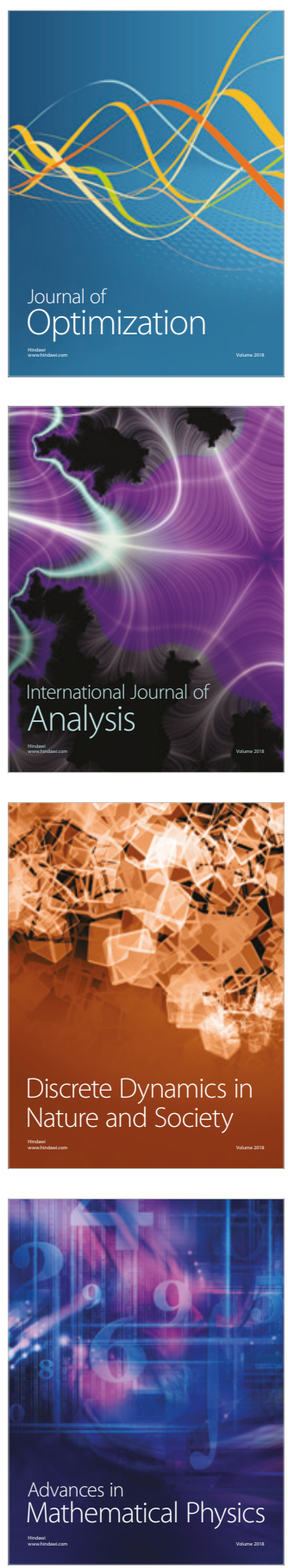